Rev. Adm. Saúde (On-line), São Paulo, v. 21, n. 82: e284, jan. - mar. 2021, Epub 16 abr. 2021 http://dx.doi.org/10.23973/ras.82.284

ARTIGO ORIGINAL

\title{
Estudo descritivo sobre o financiamento da assistência à saúde frente ao tratamento cirúrgico de fratura do tornozelo e retirada de placas/parafuso em Belo Horizonte, Minas Gerais, Brasil, 2014-2019
}

Descriptive study on health care financing for surgical treatment of ankle fractures and removal of plates / screws in Belo Horizonte, Minas Gerais, Brazil, 2014-2019.

\section{Pedro Sebastião de Oliveira Lazaroni', Luciana Alves Silveira Monteiro², Alesson Filipi Bernini ${ }^{3}$, Gustavo Damazio Heluy ${ }^{4}$}

1. Médico ortopedista e traumatologista. Departamento de ortopedia e traumatologia, Hospital Maria Amélia Lins. Fundação Hospitalar de Minas Gerais. Belo Horizonte, Minas Gerais MG

2. Enfermeira, Centro de Terapia Intensiva do Hospital das Clínicas da Universidade Federal de Minas Gerais, Belo Horizonte, Minas Gerais, MG

3. Médico ortopedista e traumatologista. Departamento de ortopedia e traumatologia, Prefeitura de Uberlândia, Uberlândia, Minas Gerais MG

4. Médico ortopedista e traumatologista. Departamento de ortopedia e traumatologia, Hospital Maria Amélia Lins, Fundação Hospitalar de Minas Gerais, Belo Horizonte, Minas Gerais, MG

\section{RESUMO}

Objetivo: descrever as internações e os custos hospitalares por tratamento cirúrgico de fratura do tornozelo unimaleolar; por tratamento cirúrgico de fratura do tornozelo bimaleolar, trimaleolar, da fratura luxação do tornozelo; e por retirada de placa e/ou parafusos, no período de 2014 a 2019, no município de Belo Horizonte, Minas Gerais. Métodos: estudo retrospectivo descritivo 
realizado entre 2014 a 2019 com dados epidemiológicos obtidos no banco de dados do Departamento de Informática do Sistema Único de Saúde, oriundos do cadastro da Autorização de Internação Hospitalar e dos valores, total e por serviços hospitalares, despendidos com o tratamento cirúrgico de fratura do tornozelo unimaleolar; o tratamento cirúrgico de fratura do tornozelo bimaleolar, trimaleolar, da fratura de luxação do tornozelo; e o procedimento de retirada de placa e/ou parafusos. Resultados: entre 2014 e 2019 foram registrados 13.721 procedimentos cirúrgicos em região de tornozelo, sendo gastos $R \$$ 7.889.737,75 com as intervenções invasivas. Conclusão: sugere-se a partir dos resultados desta pesquisa a necessidade de reforçar políticas públicas voltadas para a implementação de ações concretas, para à busca de melhor eficácia no processo de seleção de materiais, revisão de protocolos clínicos e cirúrgicos.

Palavra-chave: fraturas do tornozelo; fixação interna de fraturas; traumatismos do tornozelo; financiamento da assistência à saúde; ortopedia.

\begin{abstract}
Objective: to describe hospitalizations and hospital costs for surgical treatment of unimaleolar ankle fractures; by surgical treatment of bimaleolar, trimaleolar ankle fracture, ankle dislocation fracture; and by removing the plate and / or screws, from 2014 to 2019, in the city of Belo Horizonte, Minas Gerais.

Methods: retrospective descriptive study carried out between 2014 and 2019 with epidemiological data obtained in the database of the Department of Informatics of the Unified Health System, from the Hospitalization Authorization record and the values, total and for hospital services, spent on treatment surgical fracture of the unimaleolar ankle; surgical treatment of bimaleolar, trimaleolar ankle fracture, ankle dislocation fracture; and the procedure for removing the plate and / or screws. Results: between 2014 and 2019, 13,721 surgical procedures were recorded in the ankle region, with $R \$ 7,889,737.75$ being spent on invasive interventions. Conclusion: it is suggested from the results of this research the need to reinforce public policies aimed at implementing concrete actions, in order to seek better efficiency in the process of selecting materials, reviewing clinical and surgical protocols.
\end{abstract}

Keywords: ankle fractures; internal fracture fixation; ankle injuries; health care financing; orthopedics.

\title{
INTRODUÇÃO
}

A fratura do tornozelo, considerada uma das mais comuns na traumatologia ortopédica, chega a atingir incidência de 174 fraturas por 100.000 adultos - ano 1,2. Estima-se que o número de casos será ainda maior com o envelhecimento 
da população, o que torna o tratamento desta afecção muito importante tanto do ponto de vista ortopédico quanto econômico ${ }^{1,3,4}$.

No cenário nacional, observa-se que os acidentes automobilísticos, principalmente os relacionados às motocicletas, são os que levam a mais danos potenciais na região do tornozelo, visto que a alta energia envolvida neste tipo de trauma proporciona padrões de fraturas cada vez mais complexos 5,6 .

No âmbito mundial, nota-se que o trauma alcança estratos de incidência em condições semelhantes ao de uma pandemia, pois alcança 5,8 milhões de mortes a por ano, de modo a ser considerado uma das cinco causas mais importantes de morbi mortalidade em indivíduos com idade abaixo de 60 anos ${ }^{7}$ e a oitava causa segundo a OPAS/OMS (2018) ${ }^{8}$.

Nos Estados Unidos da América, o trauma se tornou uma das principais causas de incapacidade e óbito, principalmente entre o público infantil e de adultos jovens, com idade inferior aos 45 anos, onde o trauma é responsável por 79.000 óbitos anuais, e este total está acima das mortes por doenças não transmissíveis (49.000) e doenças infecciosas (15.000) ${ }^{9}$.

Tendo em vista a maior acurácia dos exames de imagem disponíveis atualmente, observa-se maior frequência de lesões que atingem a sindesmose tibiofibular ${ }^{10}$. Nesse sentido, o tratamento desta lesão atualmente pode ser realizado por diversos meios, como endobotons e reconstrução com enxertos 10,11. Contudo, o uso de parafusos intertibiofibulares é considerado, atualmente, a técnica mais popular entre os cirurgiões ortopédicos, seja pela ampla disponibilidade de material, seja pela menor exigência técnica ${ }^{10-12}$.

Apesar de corriqueiro, o uso dos parafusos intertibiofibulares têm um importante inconveniente que é a necessidade de um segundo ato cirúrgico para sua retirada, posto que o dispositivo é uma fixação rígida intra-articular na membrana interóssea, aumentando assim os gastos com o financiamento da assistência à saúde no que se refere aos procedimentos ortopédicos e, por conseguinte, o risco para o paciente ${ }^{13}$.

Nesse sentido, torna-se importante avaliar o impacto econômico que o procedimento de retirada de parafuso intertibiofibular, para que se possa comparar com maior clareza o uso deste método com outros que não exigem uma segunda cirurgia, estabelecendo melhor estratégia de custos para os sistemas de saúde, bem como permitir traçar outras estratégias com custo menor e mais eficazes para os pacientes como uso de materiais que não necessitam de retirada.

O presente estudo tem por objetivo descrever as internações e os custos hospitalares por tratamento cirúrgico de fratura do tornozelo unimaleolar; por tratamento cirúrgico de fratura do tornozelo bimaleolar, trimaleolar, da fratura luxação do tornozelo; e por retirada de placa e/ou parafusos, no período de 2014 a 2019, no município de Belo Horizonte, Minas Gerais. 


\section{MATERIAIS E MÉTODOS}

Estudo retrospectivo descritivo realizado entre 2014 a 2019 com dados epidemiológicos obtidos no banco de dados do Departamento de Informática do Sistema Único de Saúde (DATASUS) ${ }^{14}$, oriundos do formulário "Autorização de Internação Hospitalar" (AlH), documento preenchido pelo médico responsável no momento da internação hospitalar, bem como de dados referentes aos valores total e por serviços hospitalares despendidos com o tratamento cirúrgico de fratura do tornozelo unimaleolar (código 0408050578); o tratamento cirúrgico de fratura do tornozelo bimaleolar, trimaleolar, da fratura de luxação do tornozelo (código 0408050497); e o procedimento de retirada de placa e/ou parafusos (código 0408060379).

Foram incluídos os dados referentes a pacientes com idade superior a 18 anos, cujo diagnóstico primário de internação contemple os códigos mencionados. As estimativas populacionais foram obtidas a partir dos censos demográficos do Instituto Brasileiro de Geografia e Estatística (IBGE).

Considerando que o referido estudo se baseou em um banco de dados de domínio público, sem dados de identificação, o estudo foi dispensado de apreciação por Comitê de Ética e Pesquisa conforme disposto na resolução número 466 do Conselho Nacional de Ética em Pesquisa ${ }^{15}$.

\section{RESULTADOS}

De acordo com os dados extraídos do DATASUS ${ }^{14}$, nos anos 2014 a 2019 foram registrados 49.262 procedimentos com os códigos 0408050578 , 0408050497, 0408060379, sendo que 14.657 procedimentos cirúrgicos para o tratamento de fratura do tornozelo unimaleolar; 17.315 referente às cirurgias para tratamento de fratura do tornozelo bimaleolar, trimaleolar, da fratura de luxação do tornozelo; e 17.290 para retirada de placa e/ou parafusos em todo o estado de Minas Gerais.

Considerando apenas o município de Belo Horizonte, percebe-se que, no mesmo período, o número de cirurgias para tratamento de fratura do tornozelo unimaleolar foi de 4.137 procedimentos; considerando o tratamento cirúrgicos para o tratamento de fratura do tornozelo bimaleolar, trimaleolar, da fratura de luxação do tornozelo evidenciou-se 3.967 procedimentos; e - finalmente foram detectados 5.617 registros no DATASUS referente ao procedimento de retirada de placa e/ou parafusos, perfazendo um total de 13.721 procedimentos relacionados ao tornozelo realizados nas instituições hospitalares vinculadas ao Sistema Único de Saúde (SUS), sendo a distribuição por ano apresentada na tabela 1.

Tabela 1. Número de procedimentos registrados no DATASUS por meio de código de procedimento contido em Autorização de Internação Hospitalar (AlH) em Belo Horizonte, MG, no período de 2014 a 2019. Fonte: DATASUS. 


\begin{tabular}{|c|c|c|c|c|c|c|c|c|c|c|c|c|}
\hline \multirow{2}{*}{$\begin{array}{l}\text { Procedimento } \\
\text { registrado na } \\
\text { AlH }\end{array}$} & \multicolumn{2}{|l|}{2014} & \multicolumn{2}{|l|}{2015} & \multicolumn{2}{|l|}{2016} & \multicolumn{2}{|l|}{2017} & \multicolumn{2}{|l|}{2018} & \multicolumn{2}{|l|}{2019} \\
\hline & $n$ & $\%$ & $n$ & $\%$ & $n$ & $\%$ & $n$ & $\%$ & $n$ & $\%$ & $n$ & $\%$ \\
\hline $\begin{array}{l}\text { Tratamento } \\
\text { cirúrgico de } \\
\text { fratura do } \\
\text { tornozelo } \\
\text { unimaleolar }\end{array}$ & 677 & 33,7 & 709 & 31,9 & 703 & 28,3 & 741 & 31,1 & 687 & 29,5 & 583 & 26,8 \\
\hline $\begin{array}{l}\text { Tratamento } \\
\text { cirúrgico de } \\
\text { fratura do } \\
\text { tornozelo } \\
\text { bimaleolar, } \\
\text { trimaleolar, da } \\
\text { fratura de } \\
\text { luxação do } \\
\text { tornozelo }\end{array}$ & 609 & 30,4 & 571 & 25,7 & 677 & 27,3 & 641 & 26,9 & 731 & 31,4 & 683 & 31,4 \\
\hline $\begin{array}{l}\text { Procedimento } \\
\text { de retirada de } \\
\text { placa e/ou } \\
\text { parafusos }\end{array}$ & 720 & 35,9 & 944 & 42,4 & 1102 & 44,4 & 999 & 42,0 & 910 & 39,1 & 906 & 41,7 \\
\hline Total & 2006 & 100 & 2224 & 100 & 2482 & 100 & 2381 & 100 & 2328 & 100 & 2172 & 100 \\
\hline
\end{tabular}

Encontram-se, na Tabela 2, o valor total, em reais, computado com gastos hospitalares por tratamento cirúrgico de fratura do tornozelo unimaleolar; por tratamento cirúrgico de fratura do tornozelo bimaleolar, trimaleolar, da fratura de luxação do tornozelo; e pelo procedimento de retirada de placa e/ou parafusos, entre os anos de 2014 e 2019.

Tabela 2. Valores totais (em $\mathrm{R} \$$ ) dispendidos com tratamento cirúrgico de fratura do tornozelo unimaleolar; por tratamento cirúrgico de fratura do tornozelo bimaleolar, trimaleolar, da fratura de luxação do tornozelo; e pelo procedimento de retirada de placa e/ou parafusos em Belo Horizonte - MG, entre os anos de 2014 e 2019. Fonte: DATASUS.

\begin{tabular}{|l|l|l|l|l|l|l|}
\hline $\begin{array}{l}\text { Procedimento } \\
\text { registrado na AlH }\end{array}$ & $\mathbf{2 0 1 4}$ & $\mathbf{2 0 1 5}$ & $\mathbf{2 0 1 6}$ & $\mathbf{2 0 1 7}$ & $\mathbf{2 0 1 8}$ & $\mathbf{2 0 1 9}$ \\
\hline $\begin{array}{l}\text { Tratamento cirúrgico } \\
\text { de fratura do } \\
\text { tornozelo } \\
\text { unimaleolar }\end{array}$ & $503.550,03$ & $517.769,61$ & $522.949,80$ & $551.967,82$ & $491.621,51$ & $427.987,93$ \\
\hline $\begin{array}{l}\text { Tratamento cirúrgico } \\
\text { de fratura do } \\
\text { tornozelo bimaleolar, } \\
\text { trimaleolar, da } \\
\text { fratura de luxação } \\
\text { do tornozelo }\end{array}$ & $474.461,68$ & $455.381,23$ & $561.306,01$ & $535.204,04$ & $593.167,96$ & $574.629,91$ \\
\hline $\begin{array}{l}\text { Procedimento de } \\
\text { retirada de placa } \\
\text { e/ou parafusos }\end{array}$ & $248.790,32$ & $310.933,33$ & $370.033,65$ & $260.274,23$ & $247.080,80$ & $242.627,89$ \\
\hline Total & $\mathbf{1 2 2 6 . 8 0 2 , 0 3}$ & $\mathbf{1 2 8 4 . 0 8 4 , 1 7}$ & $\mathbf{1 4 5 4 . 2 8 9 , 4 6}$ & $\mathbf{1 3 4 7 . 4 4 6 , 0 9}$ & $\mathbf{1 3 3 1 . 8 7 0 , 2 7}$ & $\mathbf{1 2 4 5 . 2 4 5 , 7 3}$ \\
\hline
\end{tabular}

Percebe-se que houve um gasto de $\mathrm{R} \$ 7.889 .737,75$ no que se refere ao valor total dispendido com os procedimentos em estudo. Quanto aos valores despendidos com os serviços hospitalares, nota-se que não há considerável 
diminuição, sendo utilizado um montante de $\mathrm{R} \$ 6.015 .636,58$, conforme apresentado na Tabela 3. Nesse sentido, evidencia-se que o valor de $R \$$ $1.860 .852,47$ foi utilizado para financiamento dos serviços profissionais.

Tabela 3. Valores dispendidos com os serviços hospitalares para o tratamento cirúrgico de fratura do tornozelo unimaleolar; o tratamento cirúrgico de fratura do tornozelo bimaleolar, trimaleolar, da fratura de luxação do tornozelo; e com o procedimento de retirada de placa e/ou parafusos em Belo Horizonte - MG, entre os anos de 2014 e 2019. Fonte: DATASUS.

\begin{tabular}{|l|l|l|l|l|l|l|}
\hline $\begin{array}{l}\text { Procedimento registrado } \\
\text { na AlH }\end{array}$ & $\mathbf{2 0 1 4}$ & $\mathbf{2 0 1 5}$ & $\mathbf{2 0 1 6}$ & $\mathbf{2 0 1 7}$ & $\mathbf{2 0 1 8}$ & $\mathbf{2 0 1 9}$ \\
\hline $\begin{array}{l}\text { Tratamento cirúrgico de } \\
\text { fratura do tornozelo } \\
\text { unimaleolar }\end{array}$ & $400.112,65$ & $412.454,58$ & $416.642,63$ & $439.937,68$ & $391.452,71$ & $340.453,61$ \\
\hline $\begin{array}{l}\text { Tratamento cirúrgico de } \\
\text { fratura do tornozelo } \\
\text { bimaleolar, trimaleolar, da } \\
\text { fratura de luxação do } \\
\text { tornozelo }\end{array}$ & $343.867,02$ & $332.927,77$ & $415.137,06$ & $398.170,62$ & $444.336,87$ & $432.300,71$ \\
\hline $\begin{array}{l}\text { Procedimento de retirada } \\
\text { de placa e/ou parafusos }\end{array}$ & $190.648,60$ & $226.620,10$ & $271.594,73$ & $192.603,29$ & $185.376,87$ & $180.999,08$ \\
\hline Total & $\mathbf{9 3 4 . 6 2 8 , 2 7}$ & $\mathbf{9 7 2 . 0 0 2 , 4 5}$ & $\mathbf{1 . 1 0 3 . 3 7 4 , 4 2}$ & $\mathbf{1 . 0 3 0 . 7 1 1 , 5 9}$ & $\mathbf{1 . 0 2 1 . 1 6 6 , 4 5}$ & $\mathbf{9 5 3 . 7 5 3 , 4 0}$ \\
\hline
\end{tabular}

O valor médio por AlH e internação no período de 2014 a 2019 foi o equivalente a $R \$ 619,90$ conforme apresentado na Figura 1. 


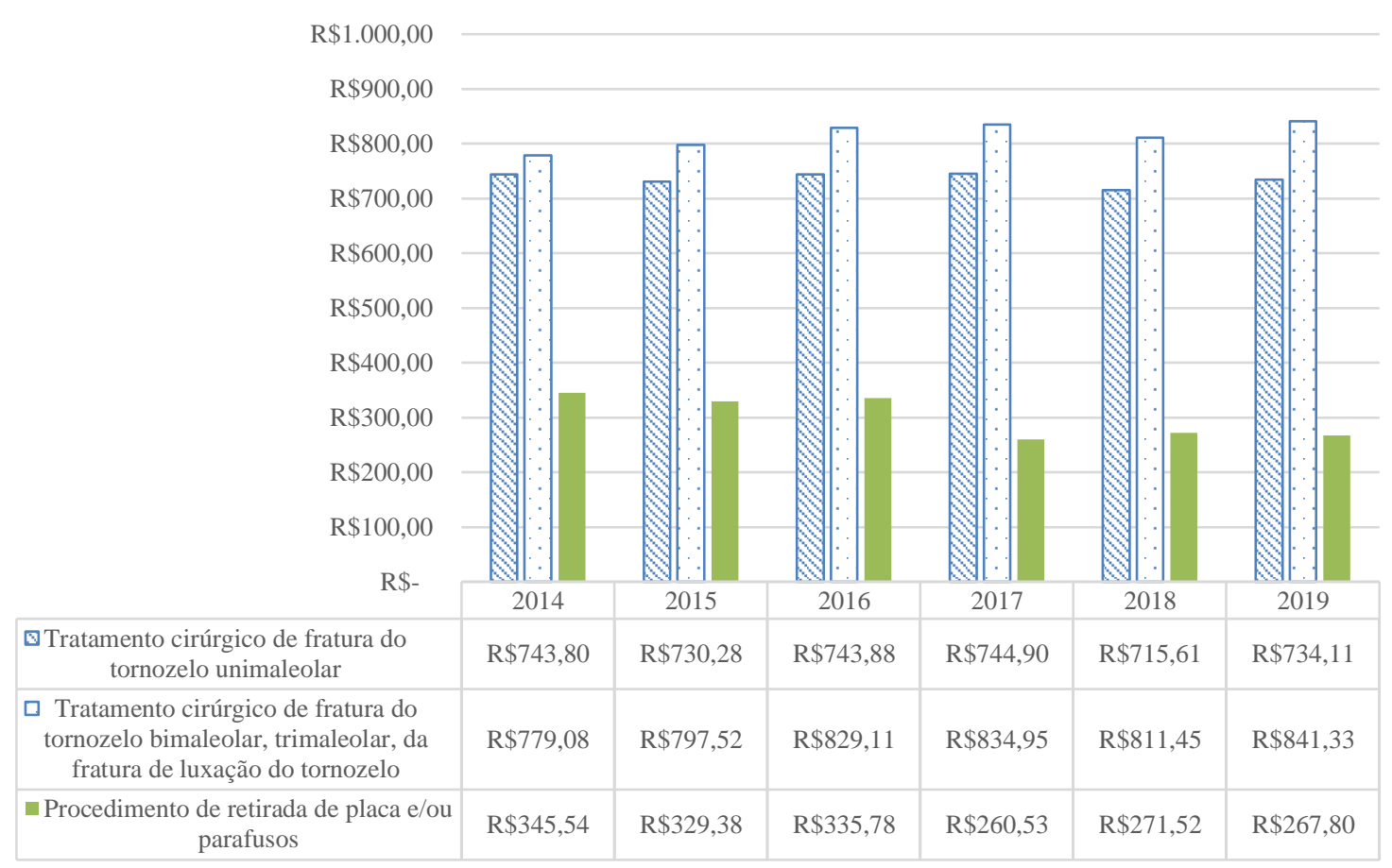

Figura 1. Distribuição do valor médio $(R \$)$ gastos com internação segundo emissão de AlH para o tratamento cirúrgico de fratura do tornozelo unimaleolar; o tratamento cirúrgico de fratura do tornozelo bimaleolar, trimaleolar, da fratura de luxação do tornozelo; e com o procedimento de retirada de placa e/ou parafusos em Belo Horizonte - MG, entre os anos de 2014 e 2019. Fonte: DATASUS

\section{DISCUSSÃO}

Considerando os dados do DATASUS ${ }^{14}$, somente no ano de 2019 , foram realizadas 47.498 cirurgias no país para tratamento cirúrgico de fratura de tornozelo no Sistema Único de Saúde (SUS), totalizando um gasto de mais de 36 milhões de reais ${ }^{16}$.

No Brasil, dois são os fatores que favorecem o aumento no número de pacientes submetidos a tratamento cirúrgico de fraturas ou fraturas-luxações do tornozelo, o aumento do número de acidente automobilísticos de alta energia e o envelhecimento da população ${ }^{17-19}$. Nesse contexto, avaliar estratégias de correção de lesões em tornozelo que favoreçam qualidade de vida no póscirúrgico, bem como uso de medidas que tentam combater o processo de diminuição da densidade mineral óssea se fazem necessárias ${ }^{18,19}$.

A análise de estratégias que apresentam melhor relação entre custo e efetividade no tratamento de lesões do tornozelo é fundamental não somente para a uma melhor recuperação dos pacientes, mas também para a viabilidade financeira dos sistemas de saúde ${ }^{20}$.

Estudos apresentam que os custos relacionados aos diferentes métodos de tratamento de lesões no tornozelo são muito divergentes, sendo ainda 
escassos os trabalhos nacionais ${ }^{16-20}$. Destaca-se que as fraturas de tornozelo demandam um grande gasto dos recursos públicos com o seu tratamento devido à sua alta prevalência e frequente necessidade intervenções cirúrgicas 19. Estudo realizado na Finlândia apresentou que o uso de materiais cirúrgicos específicos para o tratamento de fratura no tornozelo reduziu substancialmente as custas hospitalares das remoções extras, com uma redução particular nas cirurgias de remoção de placas ${ }^{20}$.

Observa-se que a maior parte dos gastos totais apresentados são decorrentes das custas com internação hospitalar, contraponto com menor valor gasto com pagamento de profissionais. Nota-se, da mesma forma, insuficiência de estudos que apresentam correlação entre as variáveis no que se refere ao tratamento cirúrgico da fratura de tornozelo. Pesquisa que analisou a tendência temporal das internações cirúrgicas pelo Sistema Único de Saúde (SUS), expõe que, para as cirurgias osteomusculares, houve uma tendência constante entre as internações cirúrgicas e o subgrupo de procedimentos ${ }^{21}$. Ao passo que para a tendência temporal da média de permanência hospitalar conforme subgrupo de procedimentos cirúrgicos, foi crescente no período de 2008 a $2016^{21}$.

Considerando estudo realizado no município de Uberlândia, Minas Gerais, MG, Brasil, os gastos totais despendidos com cirurgias realizadas em um hospital universitário federal, está diretamente relacionado ao número de procedimentos e a complexidade dos mesmos, como é o caso das especialidades cirurgia geral, ortopedia e traumatologia ${ }^{22}$.

Ressalta-se importância quanto ao questionamento de que apesar dos parafusos geralmente utilizados nas lesões de sindesmose serem o material com maior disponibilidade e de menor custo, a internação hospitalar associada ao novo procedimento cirúrgico para a sua remoção deste material, onera os sistemas de financiamento púbico de saúde, além expor o paciente a riscos inerentes ao procedimento.

A avaliação de novas estratégias, como o uso de botões de sutura, para o tratamento das lesões de sindesmose, ainda que mais dispendiosas em primeiro momento, devem ser melhor estudadas e avaliadas tanto em relação à melhores resultados clínicos, quanto aos financeiros ${ }^{23,24}$. $O$ botão de extremidade de sutura oferece vantagens potenciais, incluindo mais mobilidade anatômica da articulação, retorno mais rápido ao suporte de peso e sem necessidade de remoção do implante ${ }^{24}$.

Tem-se que o uso de dispositivos como os botões de sutura, ainda pouco populares devido ao alto custo e à falta de familiaridade dos cirurgiões, é uma opção relevante no cenário atual, visto que os estudos recentes mostram resultados clínicos satisfatórios e sem a necessidade de sua retirada num segundo momento ${ }^{24-26}$.

\section{CONCLUSÃO}


O presente estudo revelou que, de acordo com os dados do TABNET/DATASUS, Belo Horizonte corresponde a maior proporção de procedimentos realizados no que se refere ao tratamento cirúrgico de fratura do tornozelo unimaleolar (código 0408050578); ao tratamento cirúrgico de fratura do tornozelo bimaleolar, trimaleolar, da fratura de luxação do tornozelo (código 0408050497); e ao procedimento de retirada de placa e/ou parafusos (código 0408060379), no período de 2014 a 2019.

Reflete-se que os gastos relacionados aos procedimentos podem ser minimizados, conforme literatura nacional e internacional, com a possibilidade de substituição de material cirúrgico ortopédico.

Urge novas as políticas públicas considerem implementar ações concretas, voltadas à busca de uma eficácia máxima no processo de seleção de materiais e revisão de protocolos clínicos e cirúrgicos, refletindo em qualidade de vida para o paciente e otimização de financiamento. Outro encaminhamento imprescindível é o desenvolvimento de novos trabalhos, pesquisas e extensões, com a referida temática.

\section{REFERÊNCIAS}

1. Cogan C, Liu T, Toogood P. An Assessment of Normal Tibiofibular Anatomy on Lateral Fluoroscopy. Foot Ankle Int. 2020;41(7):866-869. https://doi.org/10.1177/1071100720917639

2. Debieux P, Wajnsztejn A, Mansur NSB. Epidemiologia das lesões por entorse do tornozelo diagnosticadas em pronto atendimento de ortopedia. Inst Isr Ensino e Pesqui Albert Einstein. 2019;17(4):1-5. https://doi.org/10.31744/einstein journal/2020a04739

3. Volgas D, DeVries JG, Stannard JP. Short-term financial outcomes of pilon fractures. J foot ankle Surg Off Publ Am Coll Foot Ankle Surg. 2010;49(1):47-51. https://doi.org/10.1053/j.jfas.2009.07.017

4. Smeets B, Nijs S, Nderlita M, Vandoren C, Hoekstra H. Health Care Usage and Related Costs in Fibular Plating for AO Type 44-B Ankle Fractures in a Belgian University Hospital: An Exploratory Analysis. J foot ankle Surg Off Publ Am Coll Foot Ankle Surg. 2016;55(3):535-541. https://doi.org/10.1053/j.jfas.2016.02.004

5. Bittar CK, Júnior AC, da Costa VSDA, de Freitas Pacheco AC, Ricci RL. Epidemiological profile of motorcycle accident victims in university hospital. Acta Ortop Bras. 2020;28(2):97-99. https://doi.org/10.1590/1413$\underline{785220202802230035}$

6. Bittar CK, Cliquet Junior A, da Costa VSDA, Pacheco AC de F, Ricci RL. Socioeconomic impact of motorcycle accident victims in the emergency 
room of a hospital (part 2). Acta Ortop Bras. 2020;28(3):149-151. https://doi.org/10.1590/1413-785220202803230036

7. Păun S, Beuran M, Negoi I, Runcanu A, Gaspar B. [Trauma--epidemiology: where are we today?]. Chirurgia (Bucur). 2011;106(4):439-443.

8. Organização Pan-Americana de Saúde OM de S. 10 principais causas de morte no mundo: OPAS/OMS 2018. Published 2018. Accessed November 30, 2020.

https://www.paho.org/bra/index.php?option=com content\&view=article\&id=5 638:10-principais-causas-de-morte-no-mundo\&ltemid=0

9. Maggio $C$, Chee $P$, Shinseki M, et al. Traumatic Injury in the US. Injury. 2016;176(1):139-148. https://doi.org/10.1016/j.injury.2016.04.002

10. Tourné $\mathrm{Y}$, Molinier F, Andrieu M, Porta J, Barbier G. Diagnosis and treatment of tibiofibular syndesmosis lesions. Orthop Traumatol Surg Res. 2019;105(8S):S275-S286. https://doi.org/doi:10.1016/j.otsr.2019.09.014

11. Huang $\mathrm{H}$, Yang $\mathrm{Y}$. [Research progress in diagnosis and treatment of distal tibiofibular syndesmosis injury]. J reparative Reconstr Surg. 2020;34(10):1346-1351. https://doi.org/10.7507/1002-1892.201911090

12. Song L, Liao Z, Kuang Z, et al. Comparison of tendon suture fixation and cortical screw fixation for treatment of distal tibiofibular syndesmosis injury: A case-control study. Medicine (Baltimore). 2020;99(34):e21573. https://doi.org/10.1097/MD.0000000000021573

13. Chilmi MZ, Desnantyo AT, Widhiyanto L, Wirashada BC. Low tibial and fibular osteotomy for treating varus-type post-traumatic ankle osteoarthritis: A case report. Malaysian Orthop J. 2020;14(2):145-148. https://doi.org/10.5704/MOJ.2007.025

14. Brasil. Datasus: informações de saúde. Published 2020. Accessed November 30, 2020. https://datasus.saude.gov.br/informacoes-de-saudetabnet/

15. Brasil. Resolução no 466, de 12 de dezembro de 2012. Cons Nac Ética em Pesqui. 2012; DOU no. 12: 01-12. https://conselho.saude.gov.br/resolucoes/2012/Reso466.pdf

16. Goost H, Wimmer MD, Barg A, Kabir K, Valderrabano V, Burger C. Fractures of the Ankle Joint: Investigation and Treatment Options. Dtsch Arztebl Int. 2014;111(21):377-388. https://doi.org/10.3238/arztebl.2014.0377

17. Scheer RC, Newman JM, Zhou JJ, et al. Ankle Fracture Epidemiology in the United States: Patient-Related Trends and Mechanisms of Injury. J foot ankle Surg. 2020;59(3):479-483. https://doi.org/10.1053/.j.jas.2019.09.016

18. Silva ARB, Martinez LC, Pinheiro MM, Szejnfeld VL. Low-trauma ankle fractures in Brazil: secular trends in patients over 50 years old from 2004 to 
2013. Arch Osteoporos. 2020;15(1). https://doi.org/10.1007/s11657-020$\underline{00777-6}$

19. Rasmussen CG, Jørgensen SB, Larsen P, Horodyskyy M, Kjær IL, Elsoe R. Population-based incidence and epidemiology of 5912 foot fractures. Foot ankle Surg. https://doi.org/10.1016/j.fas.2020.03.009

20. Partio N, Huttunen TT, Mäenpää HM, Mattila VM. Reduced incidence and economic cost of hardware removal after ankle fracture surgery: a 20-year nationwide registry study. Acta Orthop. 2020;91(3):331-335.

https://doi.org/10.1080/17453674.2020.1733749

21. Covre ER, Melo WA de, Tostes MF do P, Fernandes CAM. Permanence, cost and mortality related to surgical admissions by the Unified Health System. Rev Lat Am Enfermagem. 2019;27. https://doi.org/10.1590/1518$\underline{8345.2618-3136}$

22. Sousa AFM de. Custos cirúrgicos versus repasse do SUS: conhecendo a realidade do hospital de clínicas da Universidade Federal de Uberlândia. https://repositorio.ufu.br/handle/123456789/26601

23. Michelson JD, Wright M, Blankstein M. Syndesmotic Ankle Fractures. J Orthop Trauma. 2018;32(1):10-14. https://doi.org/10.1097/BOT.0000000000000937

24. Shafiq B, Thamyongkit S. Bimalleolar Ankle Fracture with Syndesmotic Sprain: ORIF and Suture-Endobutton Repair. J Orthop Trauma. 2019;33 Suppl 1:S36-S37. https://doi.org/10.1097/BOT.0000000000001538

25. Kapadia BH, Sabarese MJ, Chatterjee D, et al. Evaluating success rate and comparing complications of operative techniques used to treat chronic syndesmosis injuries. J Orthop. 2020;22(March):225-230. https://doi.org/10.1016/j.jor.2020.04.011

26. Wang W, Fan Y, Liu C, et al. [Short-term effectiveness of Endobutton plate in reconstruction of Lisfranc ligament]. J Reparative Reconstr Surg. 2020;34(11):1382-1386. https://doi.org/10.7507/1002-1892.202005034

Recebido: 30 de março de 2021. Aceito: 15 de abril de 2021 
Correspondência: Pedro Sebastião de Oliveira Lazaroni. E-mail: pedro.lazaroni@gmail.com

Conflito de Interesses: os autores declararam não haver conflito de interesses.

(C) This is an Open Access article distributed under the terms of the Creative Commons Attribution License, which permits unrestricted use, distribution, and reproduction in any medium, provided the original work is properly cited 